\title{
Proceeding
}

Supplementary Issue: Winter Conferences of Sports Science. Costa Blanca Sports Science Events, 24 April 2020. Alicante, Spain.

\section{The use of the APCM-2 protocol for a preventive assessment of the motor coordination disorder}

\author{
ROSANNA PERRONE, LUCIA PALLONETTO , CARMEN PALUMBO \\ Department of Human Sciences, Philosophy and Education, University of Salerno, Italy
}

\begin{abstract}
The development of motor skills that occurs gradually in a child's body and culminates around 8-9 years, if it occurs correctly, is the element that allows him to explore and take measures to interact with the surrounding reality. (Le Boulche, 1988) Only at this age we can speak of the "ability of space-time coordination", which takes shape and materializes only through the experience of consecutive motor acts with which they represent themselves, programmed in a time scan. (Piaget, 1976) These motor experiences are fundamental, because if the child has difficulty to complete the temporal scan, it would mean that he is the bearer of a movement disorder, albeit minor, called dyspraxia which without the appropriate tools, allowed by the following test could not be diagnosed. (Gargano, 2013) It was conducted A field research through the APCM2 protocol (Sabbadini, 2018) on a sample of 281 children. Through it, we isolated 34 subjects with specific areas that were found to be deficient, therefore more compromised by suspected dyspraxia. The most important data that emerged was that the dyspractic disorder can be overcome only if identified with a timely evaluation that allows a rehabilitation program applied as quickly as possible. (Risoli, 2016) This is important because this disorder, even if defined as "minor", in the most severe cases is influential on school performance and can sometimes lead to the boy who is affected some difficulties in simple daily motor actions.
\end{abstract}

Keywords: Dyspraxia; APCM-2 protocol; Diagnostic prevention.

\section{Cite this article as:}

Perrone, R., Pallonetto, L., \& Palumbo, C. (2020). The use of the APCM-2 protocol for a preventive assessment of the motor coordination disorder. Journal of Human Sport and Exercise, 15(2proc), S232-S243. doi:https://doi.org/10.14198/jhse.2020.15.Proc2.14

Corresponding author. Department of Human Sciences, Philosophy and Education, University of Salerno, Italy.

E-mail: Ipallonetto@unisa.it

Supplementary Issue: Winter Conferences of Sports Science. Costa Blanca Sports Science Events, 24 April 2020. Alicante, Spain.

JOURNAL OF HUMAN SPORT \& EXERCISE ISSN 1988-5202

(c) Faculty of Education. University of Alicante

doi:10.14198/jhse.2020.15.Proc2.14 


\section{INTRODUCTION}

Through the various motor experiences lived through the body and with the body, the child can access the various stages of mental development, overcoming the affective and imaginary world and entering an increasingly objective reality. In this way the movement is first mentally processed and then performed. For this reason, motor skills can be defined as the fundamental support for human relationships and activities (Gomez,2004). Children use the body as an instrument of self-knowledge from birth and in this way, they also become aware of the world around them.

Movement represents the first factor of knowledge and learning: discovering, jumping, searching, playing, running at school, playing with your body, promotes well-being and psycho-physical balance. In addition, the control of the body also allows to experiment own potential and the limits of own body, avoiding risky movements (Miur,2012). In the child, to foster global growth, two forces must coexist that act simultaneously on both his perceptions and his emotions, and on the body, because his curiosity feeds on precise movements. In his daily activities, such as running, jumping, and other basic motor patterns, the child is able to acquire new fundamental knowledge for his development and autonomy.

A healthy motor education can guarantee the improvement of intellectual work skills, which is why this subject should be considered fundamental in the basic training of every man. It is therefore important to impose a motor skills education that helps the individual to gradually dominate and coordinate his movements, thereby also promoting the development of psychic life. If we fight or prevent the psychophysical disorganization that could occur in the years of childhood and childhood by resorting to an adequate motor education, we can strengthen your self-awareness, balance emotions and impulses, reorganize our relationships with the surrounding environment (Gomez,2004).

In what are the so-called "Stages" for the achievement of the body pattern we find: the first, that is the "lived body", conventionally reached around 3 years, the second in which the discrimination of the senses seems to be clear, therefore perceptive, achieved from 3 to 6 years, and finally a third stage, which can be defined as the last, identified as "stage of the body represented", from 7 to 12 years. In the first two phases, motor behaviour can be considered global type, in which the child is dedicated to proceeding by "trial and error" by acquiring new movement praxis and new skills, also through the imitation of the adult; he will then begin gradually to perceive his body within a given time and space. The third stage, which can be traced back to Piaget's "stage of concrete operations", which enjoys those peculiarities that characterize multiple motor actions, with mastery of the movements that compose them, and with the possibility of creating motor programs taking into account the set of proprioceptive and exteroceptive information, respecting the temporal scans in relation to the context (Le Boulch,2017).

The image that a child can have of his own body can be achieved through a global knowledge of the same, which tries to analyse it in both the static and dynamic positions that it can take and above all in relation to space and what makes up its external reality. In the idea that we have of what is called the body scheme, it is to be understood how this relationship with the body does not develop immediately but gradually, to culminate around $11-12$ years.

Having a poorly structured self-image involves possible impairments regarding the relationship that the child has with his world, especially with regard to perception, motor skills and relationship with others. 
Consequently, the child could develop deficits in space-time coding and decoding, clumsiness and uncoordinated movements and inconsistent attitudes (dyspraxia), to which problems can be added in the emotional and emotional sphere. (Le Boulch, 2017)."The term congenital dyspraxia or developmental dyspraxia (also known as Developmental Dyspraxia (DD), or Specific Developmental Disorders of Motor Function (SDD-F) or Disorders of Attention and Motor Performance (DAMP) or Developmental Coordination Disorders $(D C D)$ or disorder evolutionary coordination) is described in ICD-10 as a disorder that has difficulties with coordination, since birth and which is not attributable to other sensorineural or neuromotor deficits; it is found with a delay in the acquisition of motor development stages, sometimes accompanied by delay in language development, clumsiness in movements and delays in organizing play and drawing, school difficulties and socio-emotional and / or behavioural problems. "(Sabbadini, 2005). The praxis are all those intentional activities that allow you to act on the outside world by building motor structures, therefore, a succession of movements implemented with respect to a purpose.

The complex of actions aimed at a specific need, made precisely by motor praxis, presumes a succession of well-structured phases: preparation, composition and automation; in this way you will first have a motor control of the action in progress, then in the motor act you will acquire greater definition and coordination, until you get to an action performed in a fluid and conscious manner with little margin of error.

It is thanks to increasingly sophisticated neuroradiological investigations if to date we recognize neurological aspects that unite people with dyspraxia, in particular, periventricular hypodensities of the white matter and micro-lesions of the same are highlighted, as well as thinning of the posterior part of the corpus callosum (Sabbadini, 2005).

Dyspraxia is found mainly in premature, immature and / or low weight subjects, which in some cases could be diagnosed as secondary dyspraxia because associated with major neurological pathologies (Sabbadini, 2005).

Dyspraxia can be present in other disorders, be they learning, language or behaviour. It is necessary to know the possible comorbidities existing in order to succeed in the evaluation not to confuse the various expressions of dyspraxia and to be able to understand the various problems.

As regards specific learning disorders on a dyspractic basis, it is possible to identify them by considering various factors and understand how to distinguish a normal calligraphic profile from a dysgraphic one. In light of these problems it is necessary that the assessment in terms of dysgraphia is aimed at investigating not only the morphology of the child's handwriting, but that the assessment is also and above all concerned with investigating some specific areas in order to obtain a complete diagnostic picture and reliable:

- Motor development and motor coordination,

- Sense-motor aspect,

- Visual ocular-motion aspect,

- Visual-constructive aspect,

- Praxic-constructive aspect,

- Sequentially and gestures.

The dyspractic child will tend to make movements with his whole body, for example by tilting an arm, resting the trunk on the bench and taking on particular mimic expressions, also a sign of a deficit in the inhibition. The act of writing refers to a praxic domain, whose praxic components appeal to eye-hand coordination for the production of certain graphemes. The movement of writing requires a complex of movements including 
adduction abduction, extension, and finger abduction, along with a control of the position of the arm. The dysgraphic disorder is traced back to several aspects, but the real difficulty lies in transforming verbal information into grapheme by requiring visual-motor skills. Dyspraxia of ocular-motion implies a deficit affecting the gaze in the act of looking horizontally and vertically, and consequently disturbances result from disorders related to the ability to learn and explore, to the space-time organization, to eye-hand coordination (Gargano, 2013; Sabbadini,2005).

\section{MATERIAL AND METHODS}

The quali-quantitative field study was projected towards the use of the APCM-2 protocol to investigate and evaluate motor and praxic development, to demonstrate how useful it is to subject this scientific instrument in developmental age, both to subjects and have already carried out or that on the contrary have never carried out psychomotor evaluations.

Therefore, after these considerations, through the aforementioned APCM-2 protocol it is possible to identify the most deficient functional areas of each individual subject and demonstrate how scientifically valid this protocol can be to make a first diagnosis of any dyspraxia.

In respect of the teachers, the children and their families, the administrations were carried out in the curricular hours, requiring 90 to 100 minutes per class, and using the same classroom where possible, or using a larger classroom used for other activities that was equipped with a space large enough to carry out the various exercises. The administrations per class were then carried out in rotation in groups made up of a maximum of 8 people, in order to have the possibility of being able to evaluate each person's performance carefully and precisely, in order also to avoid having to repeat the required exercise again (the truthfulness of the data is inherent in having the task performed once after having explained and shown the exercise).

The tests are structured in 2 macro-areas: movement patterns and adaptive cognitive functions; for an optimization of data collection and evaluation times during administrations, evaluation grids have been drawn up. Specifically, each child was assigned a number and then reported in the grids on each page, so each class examined had the dossier of 11 grids, hand-filled during administrations, where each test was scored of 0 , if the test had not been performed or if it had been performed incorrectly, of 1 if the test was performed partially and of 2 if the test was performed correctly.

\section{Sample}

The sample consists of 281 children, aged between 6 and 8, divided as follows: 211 children from the institution (1) and 70 from the institution (2). The administration turned to the first and second classes.

\section{Instruments}

The exercises (provided for by the protocol) submitted to the children are listed below by items, and some of them have an explicit performance and evaluation value.

\section{Equilibrium}

The exercises (provided for by the protocol) submitted to the children are listed below by items, and some of them have an explicit performance and evaluation value.

\section{Balance}

1. Walk on tiptoes, 
This test suggests an unnatural walk, and it is precisely in such cases where balance, postural and muscle abnormalities can be found

2. Stand on one leg keeping the other bent for 10 ,

3. Jump inside a square with feet together,

4. Jump on one foot from one square to another, Making a move by using only one foot, trying not to lose balance and above all to complete the task, is a sign of an important evolutionary step in the ability to control and coordinate motor actions.

5. Walk over a line with heel-toe contact,

6. Cross: one leg and the opposite arm forward and the other leg and the other arm back.

\section{Eye movements}

1. Fixing of an immovable object placed frontally,

2. Fix and chase a moving object (with your head free),

3. He fixes and follows with his eyes a moving object with his head still in his hands,

4. Schematic movements on command (saccadic),

5. Autonomous schematic movements,

6. Autonomous rotary movement,

The child is asked to make eye and clockwise rotation movements.

7. Spontaneous movements and exploration of the environment.

Sequential movements of the hands and fingers

1. "Diadococinesi"(pronation and supination of the hands) and rotation of the wrists,

2. Option of the fingers,

3. Opposition of fingers II,

4. Pianotages,

You are asked to tap each finger of your hand in sequence on a plane.

5. Pianotages with questions.

The Pianotage test is repeated again but with the addition of a new task, during the test the child is also required to answer questions, a considerable distraction factor compared to the required motor task, a factor that could change the motor act, at least slowing down execution times.

\section{Sequencing}

1. Open and close your hands simultaneously,

2. Touch the left leg with your right hand while sitting and vice versa,

3. Jump alternating closed legs and open arms,

4. Cross with one leg back and the arm of the same side forward, and on the opposite side do exactly the opposite and try to jump more than 2 times in this way without losing the starting position; moreover, this test can be changed by adding questions to which the child will have to answer at the same time as the task is done,

5. Crusader jump with questions,

6. and 7. Sequences of geometric figures,

They consist in the elaboration of two tests which provide for the reproduction of sequences of geometric figures first in a shifted order, then in reverse order, following the placed models, and if the child does not understand, the operator can position the first figure by giving an input initial.

Dynamic coordination

1. Walk to a goal, 
2. and 3. Kick the ball with a short run and then first run and kick the ball already in motion,

3. Skip the rope.

Motor and motor skills

1. Copy of figures,

2. Griffon ages: proof that consists in copying the paths first from left to right and then from right to left of the sheet.

\section{Manual skills}

1. Untie knots,

2. 2.Cut the contours of a template,

3. Grab a ball,

4. Throw a bag filled with dried legumes by centring a box,

5. Temperature a pencil,

6. 6.Tighten a bolt to a screw,

7. Palleggiare,

8. Throw a ball on the wall and shoot it at a distance of about $60 \mathrm{~cm}$.

Symbolic gestures

Reproduce mimic gestures: Ok, Viva, Pistol, Horns, Scissors, Marameo, Hitchhiking, Intentional oro-facial movements:

1. Deflate the cheeks,

2. Move your tongue in all directions,

3. Squint your eyes,

4. Soffiare,

5. Pull a kiss,

6. Pinch your lips out.

Constructive skills

1. Reconstruction of geometric figures,

2. Sticks I,

3. Sticks II,

4. Coloured cubes I,

5. Coloured cubes.

The child is asked to reproduce three-dimensional figures, and to reproduce 4 configurations. The materials used during the administrations were the following, foreseen by the same protocol:

- Scissors,

- Ball,

- Screw and bolt,

- Rope,

- Scotch tape,

- Pencil - pencil sharpener,

- Flat wooden sticks,

- White, red and green building cubes,

- Geometric figures cut out,

- Plasticized geometric figures, 
- Empty cartons and bean bags,

- Template to be cut out,

- Pen.

\section{Research plan}

The research set a main objective: to demonstrate how the APCM-2 protocol, a psychomotor screening tool, is able to diagnose suspected cases of dyspraxia. In order to demonstrate what has been expressed, in addition to carrying out the practical tests provided for by the protocol, a questionnaire was also administered to the parents, in order to obtain anamnestic data where possible, usable and comparable with the total score obtained by the single child. Thanks to this tool, created by Letizia Sabbadini, it was possible to identify 34 subjects (See Tables 2/3) with suspected dyspraxia and / or with other coordinative deficits, and for some of these it was possible to obtain cross-referenced data and research of the etiological causes that justified particularly below average scores. (Sabbadini, 2018).

\section{Data emerged}

The data shown in the evaluation grids, created to simplify and optimize the collection times, have been inserted in tables created in turn with the Excel program of the Office package, a program that is simple to use and easy to find electronically. The variance, mean and standard deviation were then calculated for each individual subject, from both institutions and from the total sample. Below is a table that contains the values of variance, standard deviation and average of each individual subject, with upstream references on the total sample.

Table 1. Variance, standard deviation and mean values of the total sample and of the individual institutes.

\begin{tabular}{llll}
\hline & Variance & Dev. Standard & Average \\
\hline Institute 1: 211 & 146.541407 & 12.10542884 & 92.7962085 \\
Institute 2: 70 & 287.284898 & 16.94948076 & 93.9714286 \\
Total sample: 281 & 181.46 & 13.47070896 & 92.22059 \\
\hline
\end{tabular}

\section{RESULTS}

This paragraph shows the results deriving from the analysis of the whole sample and the most relevant data for the validation of the hypotheses.

The graphic shows how the average values are not diametrically different from each other, this demonstrating the high validity and reliability of the instrument, the same instrument that has been used in both institutions and with the same administration methods. The differences between the two institutes in the values of variance and standard deviation, shown in the table and in the graph, differ in an important way and the reason is to be identified in the difference in the quantity of the two "micro-samples" since the Institute 1 is composed from 211 subjects, while Institute 2 from 70 .

In the Table 2, all the data of 34 subjects can be observed, which deviate significantly from the average by as much as the standard deviation indicates, about 12 points, i.e. all those values that are placed below the threshold of 80 points. 


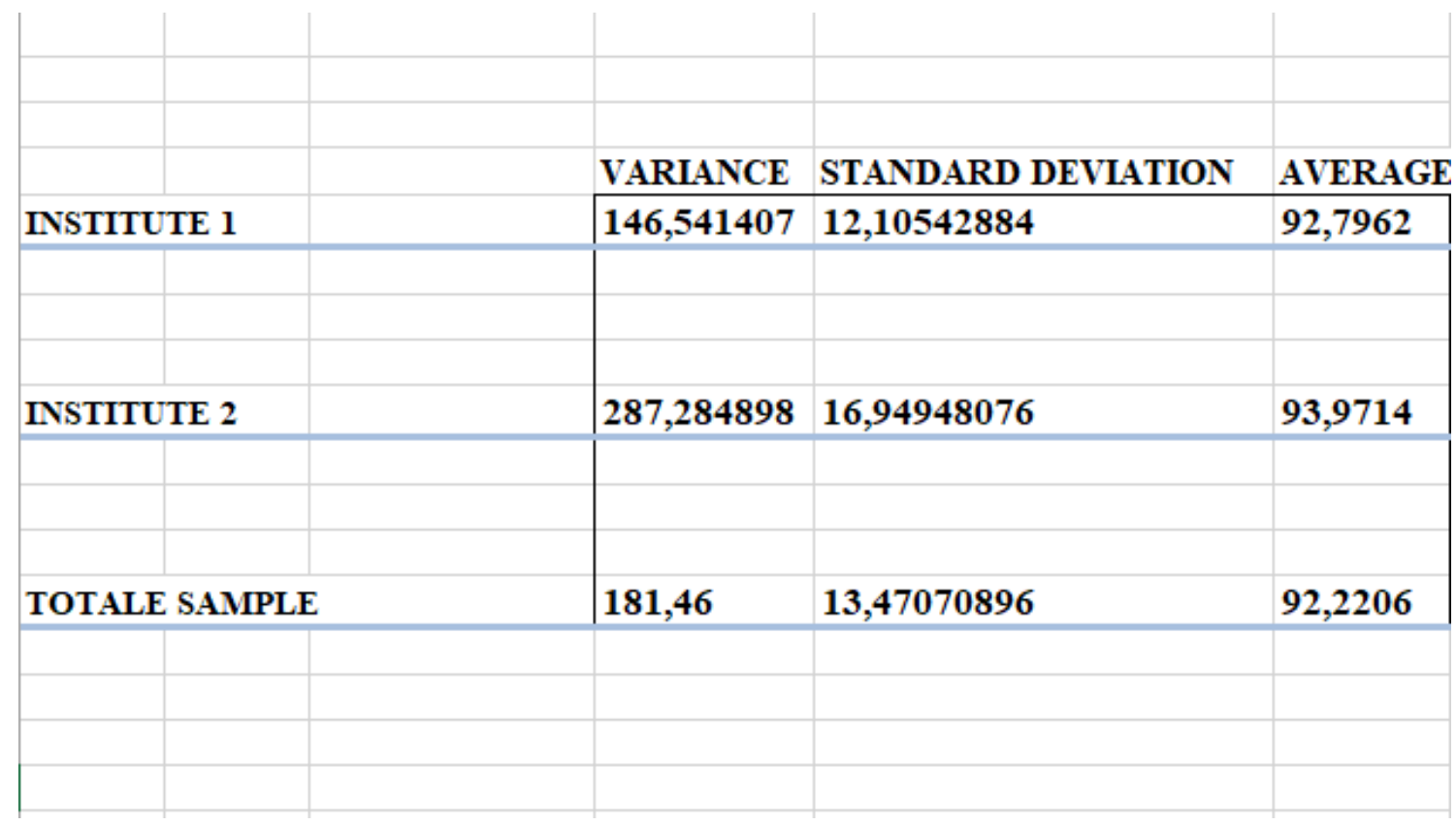

Figure 1. Excel graph with values in evidence of variance, standard deviation and average of the two single institutes and of the total sample.

\section{INSTITUTES COMPARED}

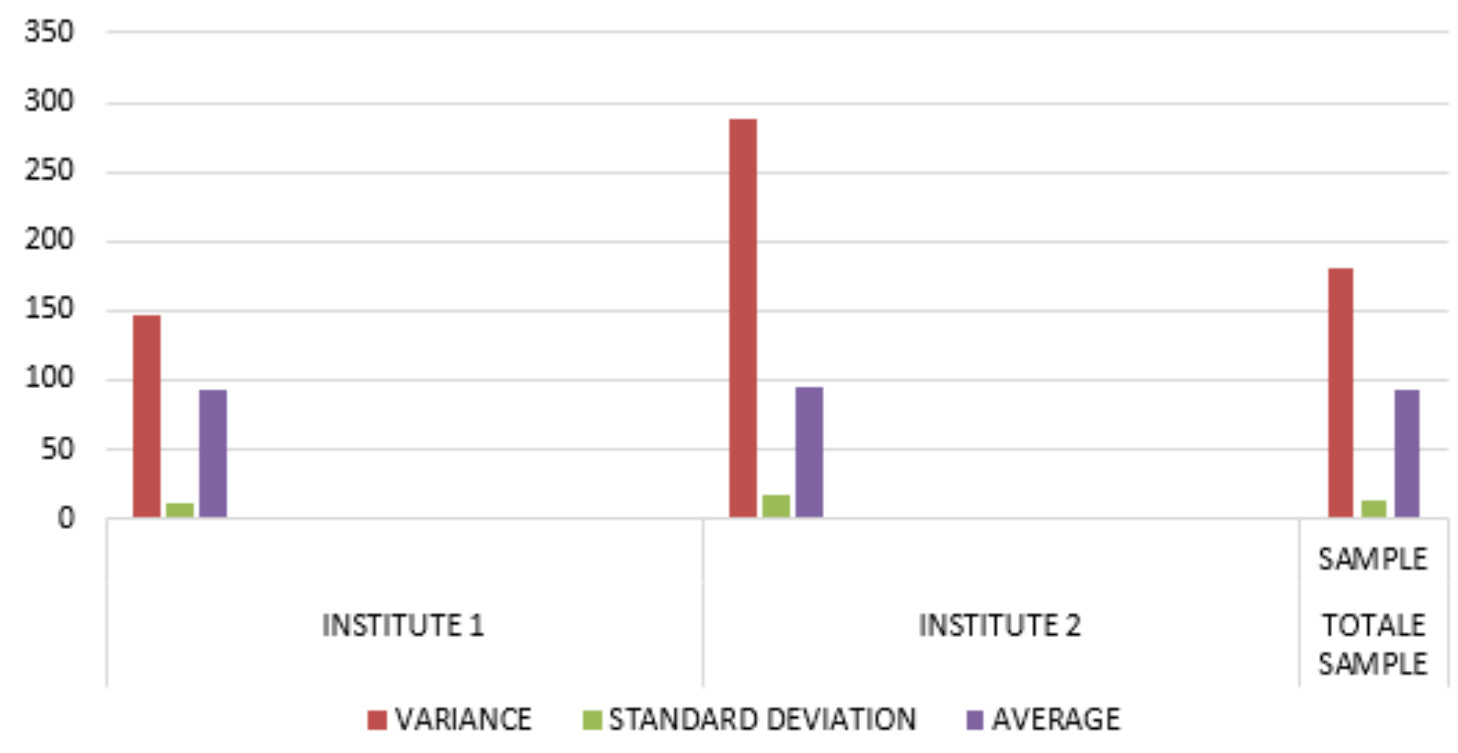

Figure 2. The bar graph shows the institutions compared to the total sample.

Table 2. The 34 deficit subjects, with a total score of less than 80 points.

Average

92.22
Dev. Stand.

13.47 


\begin{tabular}{|c|c|c|c|}
\hline Name & Tot. Score & Name & Tot. Score \\
\hline Giuseppe & 77 & Pablo & 73 \\
\hline Rosamaria & 62 & Nicola & 77 \\
\hline Andrea & 72 & Marika & 77 \\
\hline Davide & 36 & Raian & 76 \\
\hline Mariagrazia & 71 & Eros & 76 \\
\hline Daniele & 78 & Nicolo' & 68 \\
\hline Aniello & 61 & Enrico & 78 \\
\hline Doua & 64 & Francesco & 76 \\
\hline Laura & 77 & $\begin{array}{l}\text { Gaetano } \\
{ }^{*} \text { Neonatal asphyxia, difficulty to walk; }\end{array}$ & 73 \\
\hline $\begin{array}{l}\text { Rita } \\
{ }^{*} \text { Bronchial } \\
\text { asthma }\end{array}$ & 69 & Lavinia & 75 \\
\hline Angelica & 60 & $\begin{array}{l}\text { Angela } \\
\text { "Mixed developmental delay in fragile } x \\
\text { syndrome" }\end{array}$ & 67 \\
\hline Alonso & 70 & Mattias & 68 \\
\hline Adele & 76 & Michele & 75 \\
\hline Mohamed & 76 & $\begin{array}{l}\text { Vittorio } \\
\text { * Slight delay in language due to transient } \\
\text { deafness" }\end{array}$ & 70 \\
\hline Aurora & 69 & $\begin{array}{l}\text { Alfredo } \\
{ }^{*} \text { Respiratory problems at birth }\end{array}$ & 76 \\
\hline Giorgia & 77 & Luigi & 76 \\
\hline Sovaad & 60 & $\begin{array}{l}\text { Angelo } \\
{ }^{*} \text { Neonatal asphyxia, difficulty in walking }\end{array}$ & 46 \\
\hline
\end{tabular}

It is evident that not all subjects returned the completed questionnaires, it was not found, therefore being able to have qualitative data with respect to children with particular certified deficits. In the column where the names of the children are inserted, there are some boxes with an asterisk $\left(^{*}\right)$, which reports the problems concerning the subjects in question that the parents reported in the questionnaire under the items "problems at birth" and " problems in the first years of life ". It is possible and important to observe the presence of very low averages both in children who play sports and in children who do not practice it. This aspect underlines the importance of the psychomotor assessment and the tools that this growing discipline uses, specifically in this study, of the APCM-2 protocol. It would be interesting to deepen an evaluation of all the subjects present in table 2, concentrating new evaluations in the areas where there are greater difficulties and deficiencies.

Figure 3 in succession shows the subjects in question and how evident is the difference of the single score compared to the average value of about 92 points, with a score that considers the deviation as a detachment point with respect to the same average, consequently the 34 subjects all have a score that is less than 80 points. 


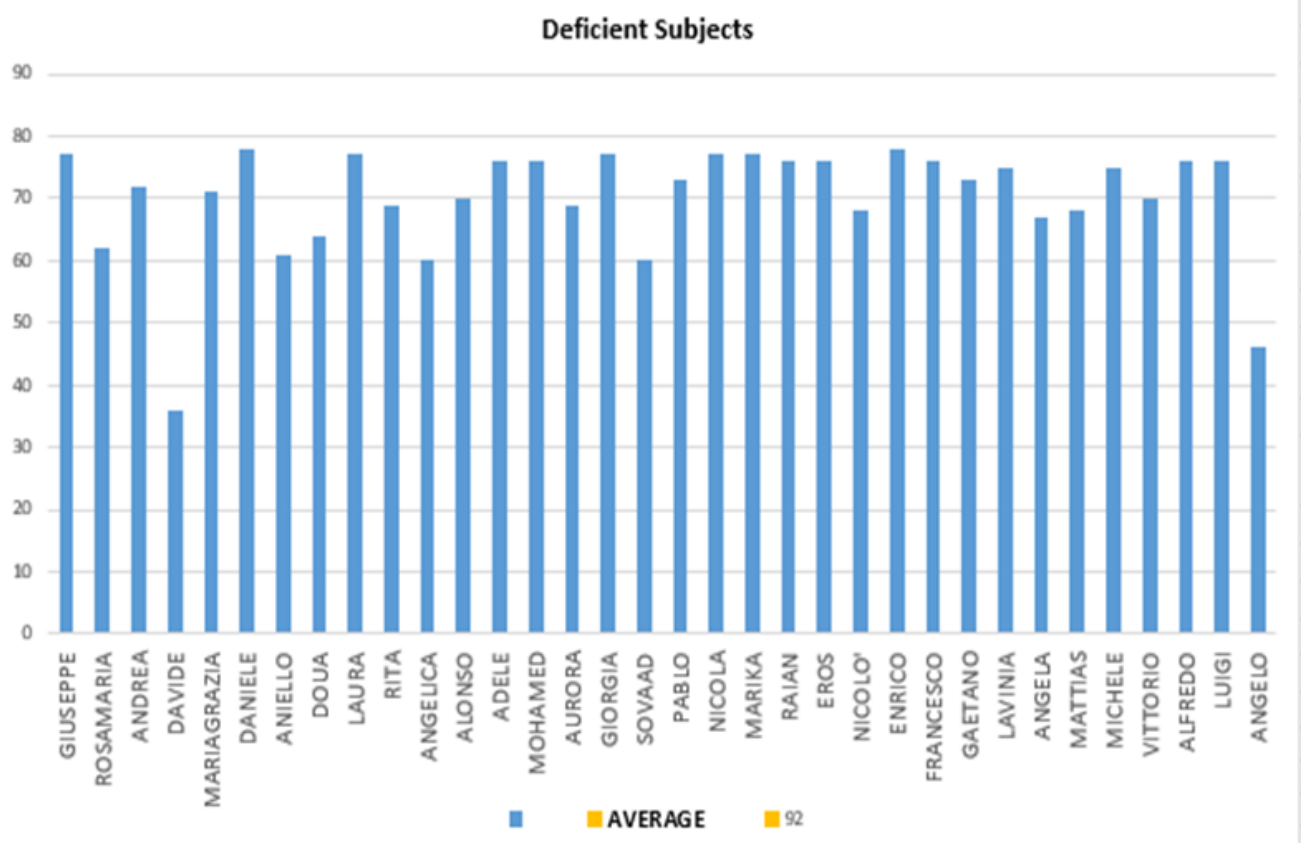

Figure 3. The graph shows the 34 subjects in relation to the average of 92 points, arranged with reference to the $Y$-axis according to the score found.

Table 3 below shows all the areas in which the subjects found greater limitations, items on which it is possible to suggest further assessment tests on the most deficient tests found by the APCM-2. Thanks to these data it is possible to understand what other probable dyspraxic deficits present in comorbidity may be.

Table 3. The items with greater impairment of the 34 subjects with suspected dyspraxia.

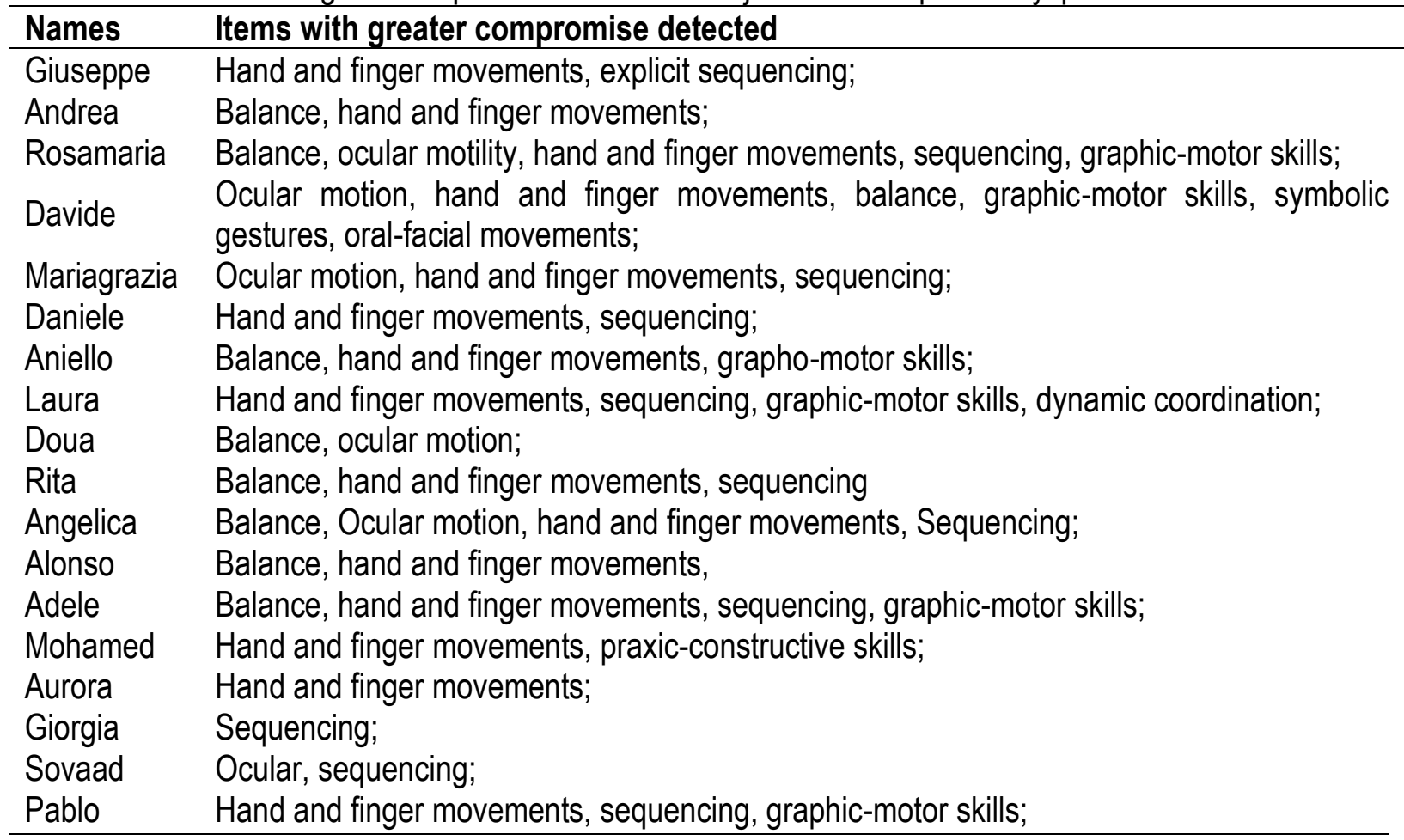




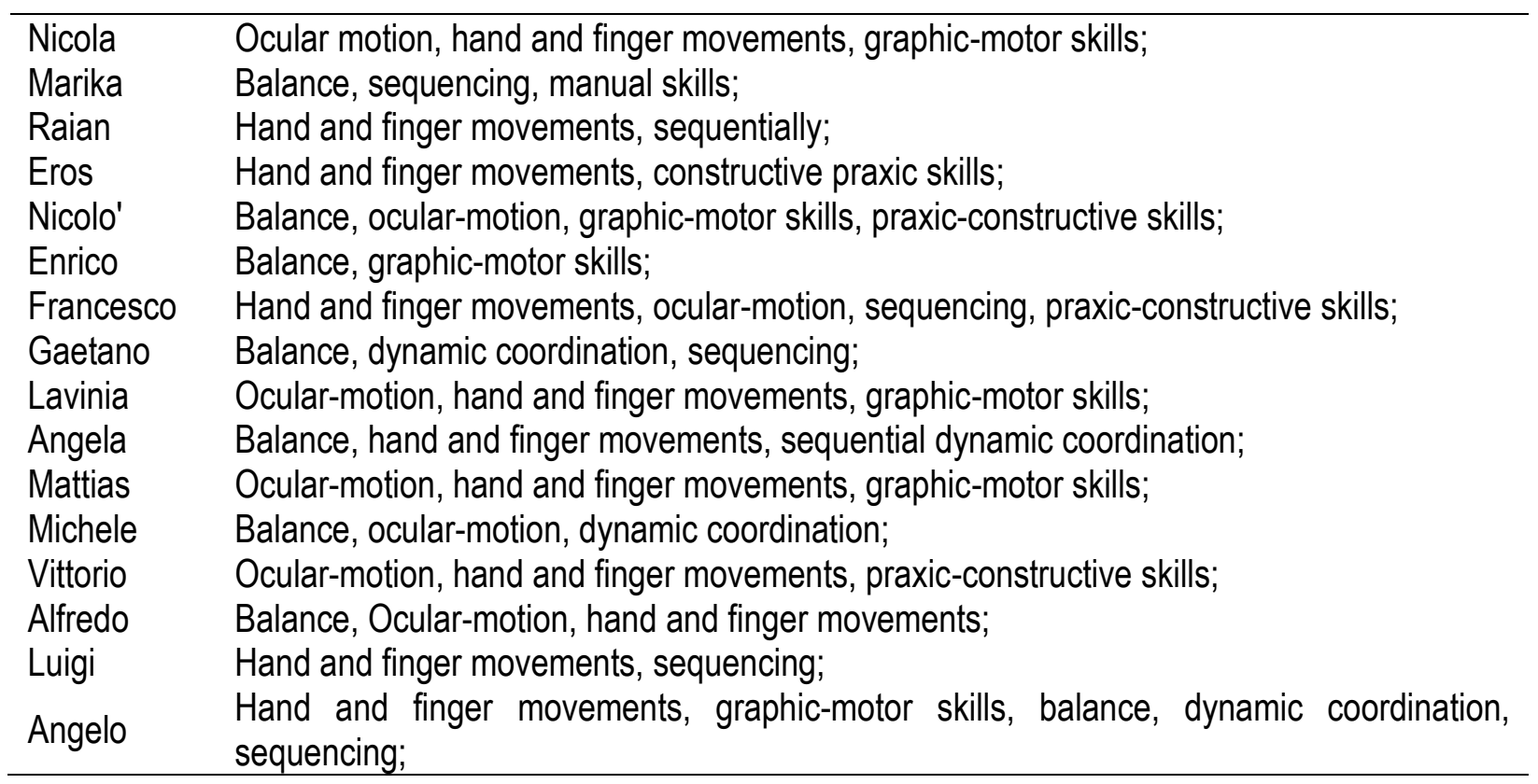

\section{CONCLUSIONS}

The final processing of the data was very interesting and satisfactory: the protocol allowed to analyse the data relating to the subjects both overall and individually, allowing an analysis of the data not only quantitative, necessary for the validation of the research itself proven on a fairly large sample, but also the observation of qualitative data.

The APCM-2 protocol allowed the identification of the real difficulties encountered by each individual in the various areas investigated, demonstrating how essential it is to use tests like this, which are able to lay the foundations for what can and must be a timely and finalized therapeutic program, which is responsive to the actual needs of the subjects, in this case of children to whom, thanks to this tool at the service of the clinician, a first diagnosis of dyspraxia, or praxic and coordination disorders can be made.

The use of the protocol has made it possible to identify specific areas of greatest compromise, and through the results obtained, we understand the importance of drawing up an early diagnosis which precedes a rehabilitation program based on a multidisciplinary intervention aimed at acquiring compromised skills, so as to guarantee an adequate functional development adapted to the different evolutionary phases.

The praxic difficulties, which can, as we have seen expressed within different items, damage and compromise the overall development of the child, this can be found in the appreciable problems in the space-time organization, in the balance and in the dynamic coordination, in the gross motor skills and in that end. Furthermore, the research has well shown how much the "neuro-diversity" pushes to make investigations, which go to evaluate on the basis of the individual person, to implement "ad personam" therapeutic plans, avoiding marginalization and being the starting point for to encourage the inclusion of dyspactic children in school and social contexts.

The APCM-2 represents a valid tool that can be used for screening projects in kindergartens, and primary schools, also to evaluate subjects already in therapy and to monitor their improvements or regressions. 
Finally, it is possible not only to diagnose specific disorders, but it is able to do it early, which is a primary and mandatory aspect in terms of prevention.

\section{AUTHOR CONTRIBUTIONS}

Perrone R. Graduated in Motor and Sport Evaluation Sciences and Analysis and Design Techniques for Disabled Sports. She is the author of the research. Pallonetto L. Ph.D. Student in Didactic Corporealities, Technology and Inclusion, at the Department of Human Sciences, Philosophy and Education of the University of Salerno, research fields: "Material and Methods". Palumbo C., Associate professor of Methods and Didactics of Motor Activities (SSD M-EDF / 01), at the Department of Human Sciences, Philosophy and Education of the University of Salerno, as Scientific Curator. Research field: "Introduction" and "Conclusion".

\section{REFERENCES}

Gargano, D. (2013). Disprassie Evolutive, Edizioni Centro Studi Erickson, Trento.

Le Boulch, J. (1988). Towards a science of human movement, Armando Editor, Rome.

Le Boulch, J. (2017). Educare con il movimento, Armando Editor, Roma.

Militerni, R. (2015). Neuropsichiatria Infantile, Idelson-Gnocchi Editore, Napoli.

Minello, R. (2013). Vygotsky and Neurodiversity. Rethinking the problems for children with Special Educational Needs. Formazione \& Insegnamento XI - 3 -2013 ISSN 1973-4778 print - 2279-7505.

Miur (2012). Indicazioni nazionali per il curricolo della scuola dell'infanzia e del primo ciclo d'istruzione, Roma, Italia.

Paloma, F. G. (2004). Corporeità ed emozioni. Una didattica psicomotoria per la costruzione del saper essere, Guida Editore, Napoli.

Piaget, J., Inhelder B. (1976). The child's conception of the space, Giunti Barbera, Florence.

Piaget, J. (1960). The praxies of the child. Rev. Neurol., pp.102-551.

Risoli, A. (2016). Disprassia in età evolutiva e riabilitazione, Carocci Editore, Roma.

Sabbadini, L. (2005). Disprassia in età evolutiva: criteri di valutazione e intervento, Springer Editore, Milano. https://doi.org/10.1007/88-470-0398-9

Sabbadini, L. (2018). Manuale APCM-2, Abilità Prassiche e della Coordinazione Motoria 2a Edizione, Hogrefe Editore, Firenze. https://doi.org/10.1007/88-470-0399-7_3 